\title{
Optimal morphokinematics for undulatory swimmers at intermediate Reynolds numbers
}

\author{
Wim M. van Rees ${ }^{1,}$, Mattia Gazzola ${ }^{2}$ and Petros Koumoutsakos ${ }^{1} \dagger$ \\ ${ }^{1}$ Chair of Computational Science, ETH Zurich, Clausiusstrasse 33, 8092 Zurich, Switzerland \\ ${ }^{2}$ School of Engineering and Applied Sciences, Harvard University, 29 Oxford Street, \\ Cambridge, MA 02138, USA
}

(Received 23 January 2015; revised 6 April 2015; accepted 13 May 2015; first published online 19 June 2015)

\begin{abstract}
Undulatory locomotion is an archetypal mode of propulsion for natural swimmers across scales. Undulatory swimmers convert transverse body oscillations into forward velocity by a complex interplay between their flexural movements, morphological features and the fluid environment. Natural evolution has produced a wide range of morphokinematic examples of undulatory swimmers that often serve as inspiration for engineering devices. It is, however, unknown to what extent natural swimmers are optimized for hydrodynamic performance. In this work, we reverse-engineer the morphology and gait for fast and efficient swimmers by coupling an evolution strategy to three-dimensional direct numerical simulations of flows at intermediate Reynolds numbers. The fastest swimmer is slender with a narrow tail fin and performs a sequence of $\mathrm{C}$-starts to maximize its average velocity. The most efficient swimmer combines moderate transverse movements with a voluminous head, tapering into a streamlined profile via a pronounced inflection point. These optimal solutions outperform anguilliform swimming zebrafish in both efficiency and speed. We investigate the transition between morphokinematic solutions in the speed-energy space, laying the foundations for the design of high-performance artificial swimming devices.
\end{abstract}

Key words: biological fluid dynamics, propulsion, swimming/flying

\section{Introduction}

Undulatory swimming is an evolutionary convergent mode for aquatic locomotion at intermediate and high Reynolds numbers. The effects of basic body kinematic properties, such as length, tail beat amplitude and frequency, on fundamental hydrodynamic performance have been captured to first order in a set of scaling laws (Gazzola, Argentina \& Mahadevan 2014). However, the hydrodynamic interplay between the swimmer's morphology and its flexural movements, although affecting hydrodynamic performance (Tytell et al. 2010), still lacks a systematic quantitative assessment.

$\dagger$ Email address for correspondence: petros@ethz.ch

$\$$ Present address: School of Engineering and Applied Sciences, Harvard University, USA. 
Observations of natural swimmers suggest biologically favourable morphokinematic combinations since specific gaits are often associated with typical morphological traits (Webb 1984). However, the evolution of swimmer shapes and gaits is not exclusively determined by hydrodynamics, as they are constrained by physiological needs as well as the properties of natural materials. Moreover, natural swimmers are able to actively employ different kinematics according to their needs, e.g. fast bursts during feeding or efficient swimming during migration (Webb 1984; Blake 2004; Kern \& Koumoutsakos 2006). Hence, it is likely that artificial swimmers specialized for single hydrodynamic metrics such as speed or efficiency will not converge to naturally existing morphokinematic solutions. Conversely, robotic swimmers have, in principle, the potential to outperform natural swimmers for specific tasks.

Numerical simulations enable direct comparisons between natural and artificial morphokinematic combinations. Computationally inexpensive low-order models of swimming bodies have been coupled to optimization techniques in Tokic \& Yue (2012) and Eloy (2013). It was found that the most efficient solutions resemble natural thunniform swimming (Tokic \& Yue 2012; Eloy 2013), whereas the fastest solutions depart from existing biological swimmers (Eloy 2013). To obtain a fundamental and detailed insight into the hydrodynamic processes associated with swimming, physically accurate direct numerical simulations are desirable. Such viscous simulations were used in Tytell et al. (2010) to highlight the intimate interplay between shape and kinematics as quantified in terms of speed and efficiency, and showed that artificial morphokinematic combinations can outperform natural swimmers.

More recently, following the work of Kern \& Koumoutsakos (2006), direct numerical simulations have been coupled to evolutionary strategies for the identification of optimal escape kinematics for a fixed morphology (Gazzola, van Rees \& Koumoutsakos 2012), and optimal morphologies for a fixed gait (van Rees, Gazzola \& Koumoutsakos 2013). The former study considered the C-start escape mechanism, a fast start consisting of a preparatory phase where the fish bends into a C-shape from rest, and a propulsive phase where the fish rapidly flips its tail to perform a swimming stroke (Domenici \& Blake 1997). It was shown in Gazzola et al. (2012) through the use of reverse-engineering and numerical simulation algorithms that this escape mechanism is qualitatively optimal for larval zebrafish morphologies. In van Rees et al. (2013), an optimization of larval swimmer morphologies showed that natural shapes are in fact suboptimal for speed and efficiency during steady anguilliform swimming. Instead, the optimal morphologies were presented and their features were analysed to explain their effects on hydrodynamic performance.

In the current study, we reverse-engineer morphology and swimming kinematics simultaneously to identify optimal solutions for undulatory swimming in terms of speed and efficiency. This generalizes our results with respect to Gazzola et al. (2012) and van Rees et al. (2013), since the current solutions can take full advantage of hydrodynamic shape/gait interplay phenomena (Tytell et al. 2010). Bearing in mind the growing potential of aquatic robotic applications (Triantafyllou \& Triantafyllou 1995; Griffiths 2003; Ijspeert et al. 2007), we disregard the unknown biological constraints that natural swimmers may be subject to. This approach enables us to systematically explore the morphokinematic landscape of undulatory swimming, without a priori (e.g. biological) assumptions and constraints on the solution space. The current work represents, to the best of our knowledge, the most ambitious inverse-design study for self-propelled swimming to date, involving over 10000 individual direct numerical simulations of 3D self-propelled bodies at intermediate Reynolds numbers that are free to adapt their gait and shapes by 15 design parameters. 


\section{Inverse design of artificial swimmers}

We perform two separate optimizations, one for maximum speed and one for maximum efficiency. In both optimizations we fix the swimmer length $L=1$ and the undulatory swimming period $T=1$. Based on these parameters we define a swimmer Reynolds number $\operatorname{Re}=\left(L^{2} / T\right) / v=550$, where $v$ is the kinematic viscosity of the fluid. The corresponding flow regime is typical of larval zebrafish at five days post-fertilization (Muller, van den Boogaart \& van Leeuwen 2008) and allows for direct comparison with previous studies (Gazzola et al. 2012; van Rees et al. 2013).

The swimmer midline kinematics is described with a curvature profile $\kappa(s, t)$, defined as an amplitude vector $\boldsymbol{K}(s)$ multiplied by a travelling wave

$$
\kappa(s, t)=K(s) \sin \left[2 \pi\left(\frac{t}{T}-\tau \frac{s}{L}\right)\right],
$$

where $s$ is the arclength, $t$ is the time and $\tau$ determines the wavelength. The amplitude vector $\boldsymbol{K}(s)$ is a cubic spline interpolating $N_{m}=6$ control points distributed at the locations $s / L=(0,1 / 20,1 / 3,2 / 3,19 / 20,1)$ along the midline (Kern \& Koumoutsakos 2006). The curvature values at these control points are denoted as $\beta_{k}^{m}$ with $k \in\left[0, N_{m}-1\right]$, where both endpoint curvatures are fixed to zero. This leads to four free parameters, $\left\{\beta_{k}^{m}\right\}_{k=1, \ldots, 4}$, for the curvature that, along with the wavelength parameter $\tau$, control the swimming gait. The 3D morphology is defined by width and height profiles described by two parameterized B-spline curves, and the $3 \mathrm{D}$ volume is constructed with elliptical cross-sections (van Rees et al. 2013). The resulting shapes are doubly symmetric and generally vary in volume. We note, however, that the volume of individual swimmers during their swimming cycles remains constant. As in van Rees et al. (2013), we use four free parameters for the width profile, $\left\{\beta_{i}^{w}\right\}_{i=1, \ldots, 4}$, and six free parameters for the height profile, $\left\{\beta_{j}^{h}\right\}_{j=1, \ldots, 6}$, resulting in a total of ten parameters determining the morphology. The morphokinematic optimization problem therefore entails a total of 15 degrees of freedom. Further details on the parameterization, and number of parameters, are discussed in the supplementary data available at http://dx.doi.org/10.1017/jfm.2015.283.

The simulations of self-propelled swimmers in an incompressible viscous flow are performed with a remeshed vortex method combined with penalization and projection techniques (Gazzola et al. 2011). The fluid-structure interaction solver is coupled with a stochastic optimization algorithm, the Covariance Matrix Adaptation Evolutionary Strategy (CMA-ES) in its multihost, rank- $\mu$ and weighted recombination form (Hansen, Muller \& Koumoutsakos 2003). As in van Rees et al. (2013), speed is evaluated by averaging the swimmer's forward velocity in the sixth swimming cycle, which we consider representative of steady swimming after the transient starting acceleration. Efficiency is defined as the ratio of useful kinetic energy to the sum of useful energy and energy delivered to the flow, again averaged over the sixth swimming cycle. Since the useful energy contains the product of the swimmer's volume and velocity, this definition may be understood as a transport efficiency. The efficiency definition and computation are discussed in more detail in the supplementary data.

We constrain the search space for kinematics and morphology to prevent unattainable solutions. For the midline kinematics the absolute curvature of each control point remains smaller than $3 \pi / L$. For the morphological parameters we impose that the resulting shapes fit within a bounding box of size $L \times 0.6 L \times 0.6 L$, as in van Rees et al. (2013). Unfeasible morphokinematic solutions involving profile curves intersecting each other or the body midline are rejected. This approach prevents the generation of irrelevant engineering as well as numerically unstable solutions, while retaining its generality given the richness of representable morphokinematic solutions. 


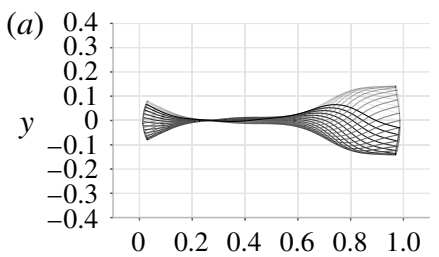

(b)

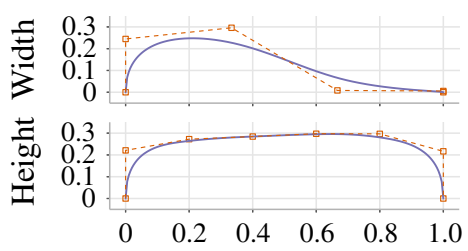

(e)

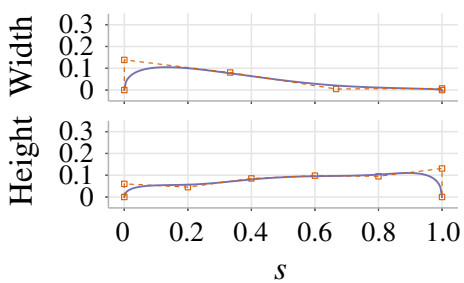

(c)

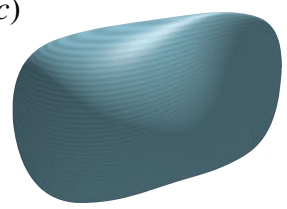

$(f)$

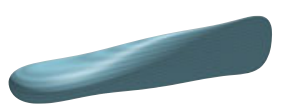

FIGURE 1. (Colour online) Optimal solutions for efficient $(a-c)$ and fast $(d-f)$ swimmers. Panels $(a, d)$ show the midline deformation during one cycle, $(b, e)$ contain the width and height profiles for each swimmer and $(c, f)$ show a 3D rendering of the optimal shapes.

\section{Optimal morphokinematics of artificial swimmers}

We optimize the morphokinematics of self-propelled swimmers with respect to the efficiency and speed metrics defined above. The optimal solutions (figure 1) are obtained after 140 generations for the efficient metric and 75 for the fast metric, corresponding to 8400 and 4500 individual simulations respectively, as detailed in the supplementary data. In this section we will discuss the morphology and kinematics of the optimal solutions; subsequently the next section will detail the corresponding swimming mechanisms from a hydrodynamical perspective.

The most efficient swimmer has midline kinematics that is reminiscent of carangiform swimming. Lateral deformations are mostly confined to the tail, combined with smaller lateral excursions at the head and almost no deformations in the middle portion of the body. The shape combines a nearly constant maximal height profile with a large width in the anterior part, sharply tapering off towards the tail via a pronounced inflection point to form a thin caudal fin.

The geometry of the fastest swimmer is characterized by a slender and narrow profile, with the height increasing and the width decreasing from the head to the tail. The anterior features are characteristic of a streamlined low-pressure-drag body, whereas the taller and thin tail resembles that of a natural swimmer in the absence of the caudal peduncle. The midline kinematics of the fast swimmer resembles that of the C-start escape mechanism. In a previous optimization study, the C-start was shown to be an optimal escape pattern for zebrafish larvae in this Reynolds number regime (Gazzola et al. 2012). The present results suggest that such a gait is also optimal for fast steady swimming, providing an acceleration burst during each cycle that sustains a high average forward velocity.

\section{Hydrodynamics of optimal artificial swimmers}

Both swimmers create lateral vortex rings in the wake, with each stroke first closing the upstream half of the previous ring and then, as the tail changes direction, shedding the downstream half of the next ring (figure 2). Due to the high speed of the fastest 
(a)

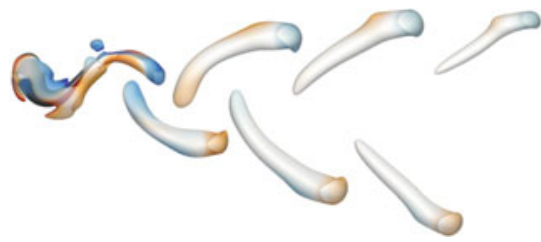

(c)

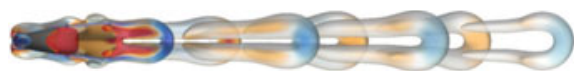

(e)

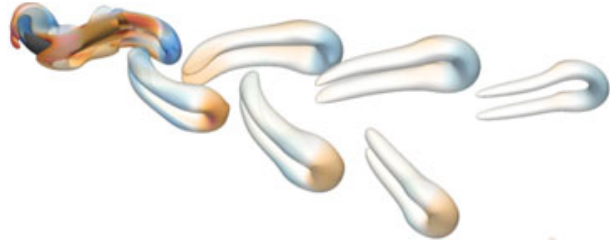

(b)

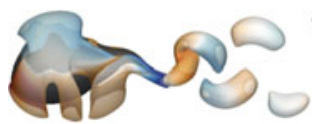

(d)

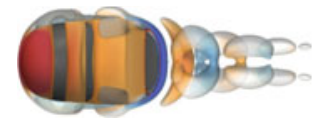

$(f)$

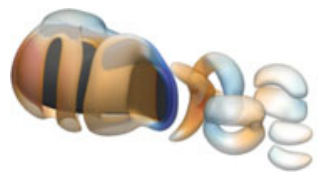

FIgURE 2. (Colour online) Visualization of the vortical structures in the wake using an isosurface of the $Q$-criterion (Hunt, Wray \& Moin 1988), coloured by the local strength of the vertical vorticity component. The swimmers are depicted in dark grey. Top view $(a, b)$, side view $(c, d)$ and perspective view $(e, f)$ of the fast $(a, c, e)$ and efficient swimmers $(b, d, f)$.

swimmer, its vortex rings are strongly elongated and resemble those of the C-start escape (Gazzola et al. 2012). The C-start mechanism relies on a strong body curvature to trap a large volume of fluid, which is accelerated backwards during the subsequent propulsive stroke so that the swimmer accelerates in the opposite direction (Gazzola et al. 2012). Each stroke of our optimally fast solution may therefore be seen as generating a burst acceleration, which cyclically repeats to sustain a high average velocity.

The vortical structures of the efficient swimmer's wake are much weaker. Due to the swimmer's lower speed and tall tail, elliptical vortex rings are shed which then converge to an almost circular configuration before their energy dissipates. The structures are qualitatively similar to those of the most efficient morphology for anguilliform swimming identified in van Rees et al. (2013). That morphology shares with our most efficient solution a pronounced inflection point in the width profile. From a hydrodynamical perspective, this is consistent with the argument that steady-state undulatory swimming relies on the same fundamental mechanism as the C-start (van Rees et al. 2013). In particular, the fluid is trapped inside convex regions on either side of the midline created by the undulatory body deformation, and accelerated backwards by the travelling wave. The inflection point located approximately midway in the width profiles of both solutions favours this mechanism, by increasing the volume of fluid that can be accommodated and accelerated backwards.

\section{Navigating the morphokinematic landscape}

To investigate the interplay between gait and morphology, we explore the morphokinematic landscape between the optimal solutions, and compare with biological swimmers. We quantify hydrodynamic performance in terms of speed and efficiency as well as useful and required energy. 

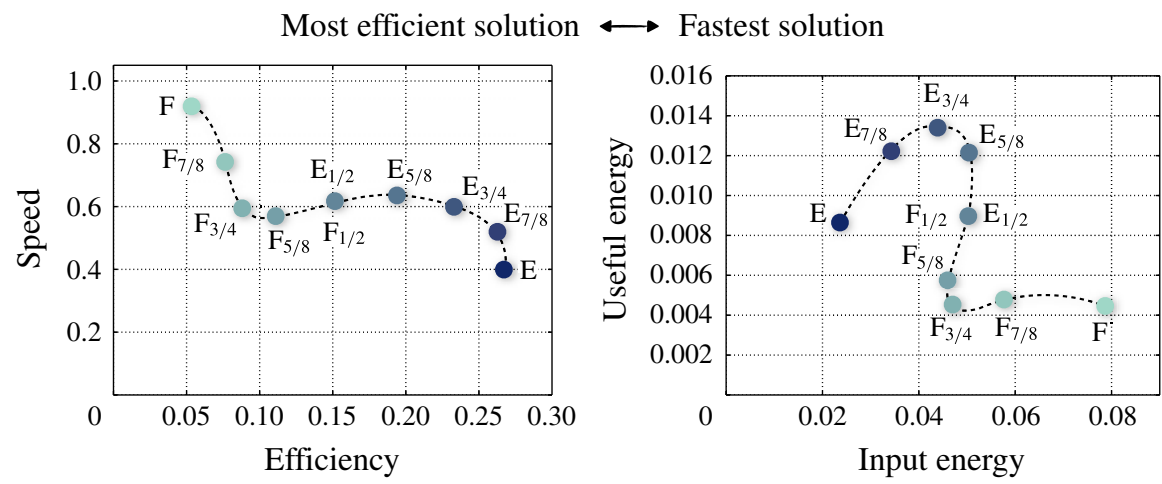

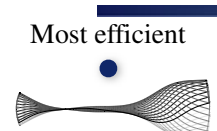

$\mathrm{E}$

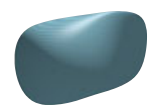

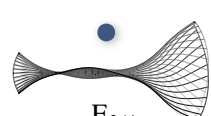

$\mathrm{E}_{3 / 4}$

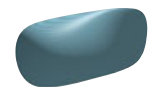

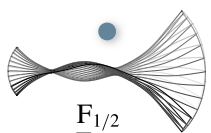

$\mathrm{E}_{1 / 2}$

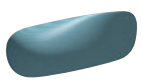

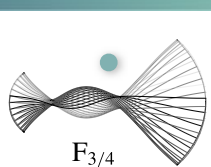

$\mathrm{F}_{3 / 4}$

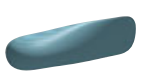

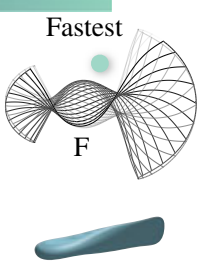

FIGURE 3. (Colour online) Results of the most efficient (E), fastest (F) and seven intermediate solutions obtained by equidistant linear combinations of the parameters of the fast and efficient swimmers. The shapes and gaits of the two optima and three intermediate solutions are provided under the plots for reference.

\section{Transition between optimal solutions}

To examine morphokinematic solutions that lie in between the fastest and most efficient solutions identified here, we simulated several swimmers constructed by interpolating all the optimization parameters between the two optimal solutions, as depicted in figure 3. We note that this approach is not meant to approximate the Pareto front between our two optimization metrics, which would be beyond the near-future capabilities of computing for the 3D direct numerical simulations of viscous flows presented herein (Bueche et al. 2002). Instead, our aim is to discuss the contribution of morphology and kinematics to one metric or another by following an intuitive path between the two optimal solutions. Comparison of the fastest swimmer (F) with its two closest hybrid solutions $\left(\mathrm{F}_{7 / 8}\right.$ and $\left.\mathrm{F}_{3 / 4}\right)$ shows how this relatively small change in shape and gait decreases the speed by approximately $35 \%$, requiring approximately $40 \%$ less energy to swim. The fastest solution therefore occupies a highly specialized place in our parameter landscape, probably requiring too much extra energy with respect to slower solutions to be biologically attainable for steady-state swimming.

On the other side of the parameter space, the second most efficient solution $\left(\mathrm{E}_{7 / 8}\right)$ still attains approximately $80 \%$ of the efficiency of the optimal case (E). It reaches this efficiency even though it is approximately $25 \%$ faster, and has approximately $40 \%$ higher useful energy, because the required energy increases correspondingly. These results imply that relatively high efficiencies can be obtained within a range of shape-gait combinations, making efficient solutions less specialized and therefore more robust than fast swimmers.

We note that the five most central swimmers $\left(E_{3 / 4}\right.$ to $\left.F_{3 / 4}\right)$ achieve approximately equal speeds with similar energetic requirements, both quantities lying between 

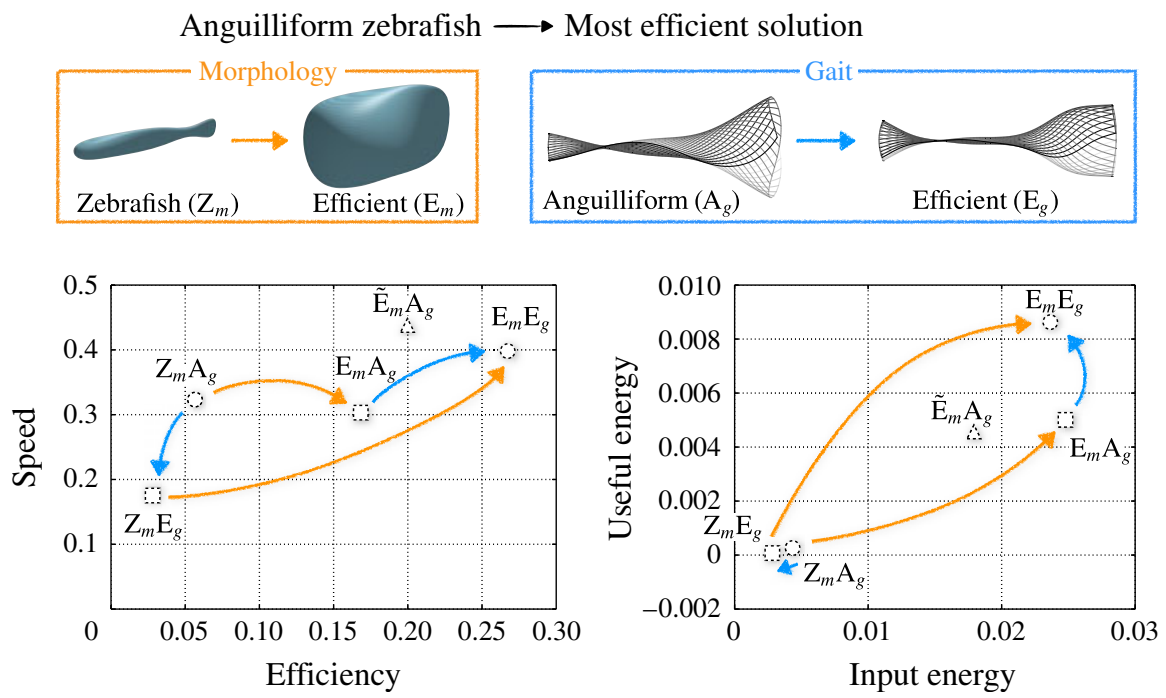

Figure 4. (Colour online) Comparison of the most efficient swimmer $\left(\mathrm{E}_{m} \mathrm{E}_{g}\right)$ with the anguilliform zebrafish $\left(\mathrm{Z}_{m} \mathrm{~A}_{g}\right)$, and two intermediate solutions interchanging the zebrafish shapes and gaits with those of the optimal solution. The triangle $\left(\tilde{\mathrm{E}}_{m} \mathrm{~A}_{g}\right)$ is the optimal shape for efficient anguilliform swimming as found in van Rees et al. (2013).

those of the fastest and most efficient solutions. This plateau in the morphokinematic landscape provides a space in which swimmer designs can be specialized for different tasks or environments, without compromising their hydrodynamic performance.

\section{Comparison with anguilliform larval zebrafish}

We compare our optimal solutions with simulations of a natural anguilliform larval zebrafish, and, as in Borazjani \& Sotiropoulos (2010), we interchange morphologies and kinematics to assess the individual contributions of shape and gait. We denote morphokinematic combinations with a two-letter sequence, using a subscript to specify morphology or gait. The combination of zebrafish morphology $\left(Z_{m}\right)$ and anguilliform gait $\left(\mathrm{A}_{g}\right)$ is thus denoted $\mathrm{Z}_{m} \mathrm{~A}_{g}$, whereas the fastest and most efficient solutions are denoted $\mathrm{F}_{m} \mathrm{~F}_{g}$ and $\mathrm{E}_{m} \mathrm{E}_{g}$ respectively. We will refer to the optimal morphologies of van Rees et al. (2013), which were optimized with the fixed anguilliform gait of the zebrafish for fast and efficient swimming, as $\tilde{\mathrm{F}}_{m}$ and $\tilde{\mathrm{E}}_{m}$. We note that since the optimal morphologies of van Rees et al. (2013) were obtained under the constraint of a fixed swimming gait, their performance is (as expected) suboptimal with respect to our current solutions obtained via a full morphokinematic optimization. In the following we discuss separately the cases of efficiency and speed, as presented in figures 4 and 5 respectively. The visual representation is complemented with table 1 , showing the numerical values of the plots normalized with respect to the anguilliform zebrafish.

Efficient swimming. For the anguilliform gait, exchanging the zebrafish shape $\left(\mathrm{Z}_{m} \mathrm{~A}_{g}\right)$ with the optimal shape $\left(\mathrm{E}_{m} \mathrm{~A}_{g}\right)$ almost triples the efficiency, as the volume is increased 20-fold whereas the steady-state speed remains almost constant. This indicates that the propulsive benefits of the larger lateral area are sufficient to compensate for the increased pressure and friction drag forces. 

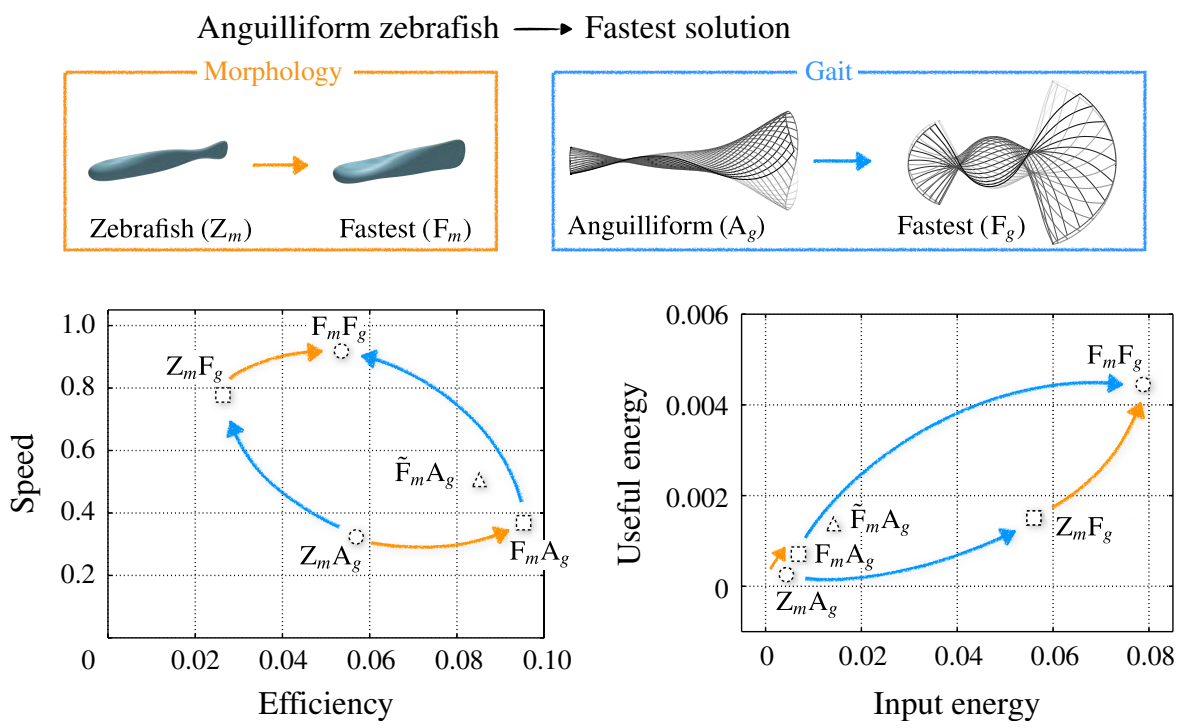

Figure 5. (Colour online) Comparison of the fastest swimmer $\left(\mathrm{F}_{m} \mathrm{~F}_{g}\right)$ with the anguilliform zebrafish $\left(\mathrm{Z}_{m} \mathrm{~A}_{g}\right)$, and two intermediate solutions interchanging the zebrafish shapes and gaits with those of the optimal solution. The triangle $\left(\tilde{\mathrm{F}}_{m} \mathrm{~A}_{g}\right)$ is the optimal shape for fast anguilliform swimming as found in van Rees et al. (2013).

\begin{tabular}{lccc} 
& \multicolumn{3}{c}{ Speed/efficiency } \\
\cline { 2 - 4 } & Anguilliform $\left(\mathrm{A}_{g}\right)$ & Fastest $\left(\mathrm{F}_{g}\right)$ & Most efficient $\left(\mathrm{E}_{g}\right)$ \\
Zebrafish $\left(\mathrm{Z}_{m}\right)$ & $1.0 / 1.0$ & $2.4 / 0.5$ & $0.5 / 0.5$ \\
Fastest $\left(\mathrm{F}_{m}\right)$ & $1.1 / 1.7$ & $2.8 / 0.9$ & - \\
Most efficient $\left(\mathrm{E}_{m}\right)$ & $0.9 / 3.0$ & - & $1.2 / 4.7$ \\
Fastest anguilliform $\left(\tilde{\mathrm{F}}_{m}\right)$ & $1.6 / 1.5$ & - & - \\
Most efficient anguilliform $\left(\tilde{\mathrm{E}}_{m}\right)$ & $1.3 / 3.5$ & - & - \\
& & Input & \\
Zebrafish $\left(\mathrm{Z}_{m}\right)$ & $1.0 / 1.0$ & $5.8 / 12.8$ & $0.3 / 0.6$ \\
Fastest $\left(\mathrm{F}_{m}\right)$ & $2.7 / 1.6$ & $17.0 / 18.0$ & - \\
Most efficient $\left(\mathrm{E}_{m}\right)$ & $19.0 / 5.7$ & - & $32.8 / 5.4$ \\
Fastest anguilliform $\left(\tilde{\mathrm{F}}_{m}\right)$ & $5.0 / 3.2$ & - & - \\
Most efficient anguilliform $\left(\tilde{\mathrm{E}}_{m}\right)$ & $17.0 / 4.1$ & - & -
\end{tabular}

TABLE 1. The values for speed/efficiency and input/useful energy of hybrid swimmers created by combining different morphologies (along the rows) with different gaits (down the columns). All values are normalized with respect to the anguilliform zebrafish. The last two rows in each part of the table correspond to the optimal morphologies for anguilliform kinematics as presented in van Rees et al. (2013).

Maintaining the optimal solution morphology and interchanging the anguilliform gait $\left(\mathrm{E}_{m} \mathrm{~A}_{g}\right)$ with the optimal gait $\left(\mathrm{E}_{m} \mathrm{E}_{g}\right)$ increases the speed by $30 \%$ and efficiency by $60 \%$, without significantly altering the energy expenditure of the swimmer. However, for the zebrafish morphology, replacing the anguilliform gait $\left(Z_{m} A_{g}\right)$ with the gait 
of the optimal solution $\left(\mathrm{Z}_{m} \mathrm{E}_{g}\right)$ decreases both the speed and the efficiency by approximately $50 \%$. An identical change in gait thus improves the performance of one morphology and degrades it for another, stressing the hydrodynamic interplay between morphology and midline kinematics in undulatory swimming.

We note that the anguilliform swimmer with the optimal solution morphology $\left(\mathrm{E}_{m} \mathrm{~A}_{g}\right)$ has only slightly lower efficiency than the most efficient anguilliform swimmer $\left(\tilde{\mathrm{E}}_{m} \mathrm{~A}_{g}\right)$, which in turn reaches already $75 \%$ of the efficiency of the optimal solution $\left(\mathrm{E}_{m} \mathrm{E}_{g}\right)$. Anguilliform midline kinematics seems therefore to be well suited for high-efficiency swimming, provided that the morphology is optimized accordingly.

Fast swimming. Figure 5 and table 1 show that when the anguilliform zebrafish $\left(\mathrm{Z}_{m} \mathrm{~A}_{g}\right)$ adopts the gait of the fastest solution, the resulting swimmer $\left(\mathrm{Z}_{m} \mathrm{~F}_{g}\right)$ reaches almost $90 \%$ of the optimal solution $\left(\mathrm{F}_{m} \mathrm{~F}_{g}\right)$ speed. On the other hand, an anguilliform swimmer with the optimal solution shape $\left(\mathrm{F}_{m} \mathrm{~A}_{g}\right)$ reaches approximately the same velocity as the anguilliform zebrafish $\left(\mathrm{Z}_{m} \mathrm{~A}_{g}\right)$. This indicates that gait rather than morphology is the predominant factor in fast swimming, correlating with the observation that fish generally can actively adjust their gait, whereas only very few can adjust their morphology. The performance increase of the fastest solution gait comes with an energetic cost, however, as the $\mathrm{Z}_{m} \mathrm{~F}_{g}$ swimmer requires 13 times more energy and sees a $50 \%$ drop in swimming efficiency compared with the anguilliform gait $\left(\mathrm{Z}_{m} \mathrm{~A}_{g}\right)$. The fastest solution gait might thus only be viable for natural swimmers during short periods of time, but could well be considered for more powerful robotic swimmers.

For the anguilliform swimming gait, we note that the shape optimized for fast anguilliform swimming $\left(\tilde{\mathrm{F}}_{m} \mathrm{~A}_{g}\right)$ is only approximately $55 \%$ faster than the anguilliform zebrafish $\left(\mathrm{Z}_{m} \mathrm{~A}_{g}\right)$, whereas the optimal shape-gait combination $\left(\mathrm{F}_{m} \mathrm{~F}_{g}\right)$ is almost $300 \%$ faster. This implies that the anguilliform kinematics is ill suited for fast swimming, even with a shape optimized for speed.

\section{Strouhal number for efficient swimming}

The reduced swimming frequency, in the context of undulatory swimming commonly denoted by the Strouhal number, is defined as $S t=f A / U$, where $f=1 / T, A$ is the lateral distance travelled by the tail during one stroke and $U$ is the averaged forward velocity. Natural swimmers typically operate at $S t \sim 0.3$, which coincides with the maximum efficiency for thrust-producing airfoils (Triantafyllou, Triantafyllou \& Grosenbaugh 1993; Taylor, Nudds \& Thomas 2003). In contrast, our most efficient swimmer has a Strouhal number of 0.72 , and a previous optimization study for efficient swimming kinematics found a Strouhal number of 0.67 (Kern \& Koumoutsakos 2006).

Here, we show that our Strouhal number values can be explained by considering the basic scaling laws of undulatory swimming (Gazzola et al. 2014), rather than the notion that hydrodynamic performance is linked to a specific Strouhal number regime. Following Gazzola et al. (2014), we have $S t \sim R e_{U}^{-1 / 4}$, where $R e_{U}=U L / v$, so that a 10 -fold reduction in the Reynolds number will double the Strouhal number. Indeed, the Reynolds numbers in this work, as well as in Kern \& Koumoutsakos (2006), are more than an order of magnitude smaller than the data in Triantafyllou et al. (1993) and Taylor et al. (2003), and the corresponding Strouhal numbers are more than a factor of two larger. We therefore conclude that the high Strouhal number of our optimally efficient swimmer is consistent with the principal scaling laws governing undulatory self-propelled swimming. 


\section{Conclusions}

We have reverse-engineered undulatory self-propelled swimmers to maximize speed or efficiency as a function of both their morphology and gait. The optimal solutions outperform natural zebrafish by over 4.5 times for efficiency or 2.5 times for speed. The fastest swimmer uses a succession of C-start swimming strokes characterized by large curvatures along the entire body, combined with a streamlined geometry profile. This result is consistent with previous studies, but may not be energetically viable for biological swimmers due to the extremely high energetic requirements. The most efficient swimmer is characterized by a large volume and carangiform-like midline kinematics, where the largest curvatures are confined to the tail and, to lesser extent, the head.

We investigated the morphokinematic landscape by examining swimmers obtained by interpolating the shape-gait parameters of the fastest and the most efficient solutions. The results show that the speed of the fastest solution is very sensitive to parameter changes, making it a highly specialized non-robust solution. Such fast swimming requires a high input energy, consistent with previous results. In contrast, the efficient swimmer is more robust to parameter deviations, as large fractions of its value can be obtained even by hybrid solutions. In between we discovered a range of swimmers with different morphokinematic characteristics but similar speeds and energetic requirements. Within this region, specialization dictated by various endogenous and exogenous constraints may take place without compromising hydrodynamic performance.

The optimal solutions identified in the present work were compared with natural anguilliform zebrafish, as well as optimal shapes for anguilliform kinematics (van Rees et al. 2013). We showed that an anguilliform swimming gait can support highly efficient swimmers, as long as the shape is suitably adapted. The actual efficiency value depends more strongly on the shape than on the gait, in the context of our definition of transport efficiency. In contrast, fast swimming relies more on the gait than on the shape, and indeed natural zebrafish larva geometries can reach $90 \%$ of the fastest morphokinematic solution speed just by adopting the corresponding C-start kinematics. We conclude that a natural swimmer could reach an impressively fast speed by suitably altering its midline kinematics, but note that the energy required to sustain this speed is up to 20 times larger than for anguilliform swimming.

Our results indicate that the present inverse-design framework is capable of identifying self-propelled swimmers that outperform biological solutions. By analysing the features of the optimal swimmers, and exploring their morphokinematic landscape, this work provides a compass for the design of high-performance artificial swimmers for engineering applications.

\section{Acknowledgements}

This work was supported by Advanced Grant No. 341117 from the European Research Council (ERC), and by the Swiss National Supercomputing Centre (CSCS) under project s436. We wish to thank the Swiss National Science Foundation (SNF) for partial financial support (WMvR, MG).

\section{Supplementary material}

Supplementary material is available at http://dx.doi.org/10.1017/jfm.2015.283. 


\section{REFERENCES}

Blake, R. W. 2004 Fish function design and swimming performance. J. Fish Biol. 65, 1193-1222. BoraZjani, I. \& Sotiropoulos, F. 2010 On the role of form and kinematics on the hydrodynamics of self-propelled body/caudal fin swimming. J. Expl Biol. 213 (1), 89-107.

Bueche, D., Stoll, P., Dornberger, R. \& Koumoutsakos, P. 2002 Multi-objective evolutionary algorithm for the optimization of noisy combustion problems. IEEE Trans. Syst. Man Cybern. C 32 (4), 460-473.

Domenici, P. \& Blake, R. W. 1997 The kinematics and performance of fish fast-start swimming. J. Expl Biol. 200 (8), 1165-1178.

EloY, C. 2013 On the best design for undulatory swimming. J. Fluid Mech. 717, 48-89.

Gazzola, M., Argentina, M. \& Mahadevan, L. 2014 Scaling macroscopic aquatic locomotion. Nat. Phys. 10, 758-761.

Gazzola, M., Chatelain, P., van Rees, W. M. \& Koumoutsakos, P. 2011 Simulations of single and multiple swimmers with non-divergence free deforming geometries. J. Comput. Phys. 230 (19), 7093-7114.

Gazzola, M., van Rees, W. M. \& Koumoutsakos, P. 2012 C-start: optimal start of larval fish. J. Fluid Mech. 698, 5-18.

Griffiths, G. (Ed.) 2003 Technology and Applications of Autonomous Underwater Vehicles. Taylor and Francis.

Hansen, N., Muller, S. D. \& Koumoutsakos, P. 2003 Reducing the time complexity of the derandomized evolution strategy with covariance matrix adaptation (CMA-ES). Evol. Comput. 11 (1), 1-18.

Hunt, J. C. R., Wray, A. A. \& Moin, P. 1988 Eddies, streams, and convergence zones in turbulent flows. Tech. Rep. CTR-S88. Center for Turbulence Research Report.

Ijspeert, A. J., Crespi, A., Ryczko, D. \& CABelguen, J. M. 2007 From swimming to walking with a salamander robot driven by a spinal cord model. Science 315 (5817), 1416-1420.

Kern, S. \& Koumoutsakos, P. 2006 Simulations of optimized anguilliform swimming. J. Expl Biol. 209 (24), 4841-4857.

Muller, U. K., VAn den BoogaArt, J. G. M. \& VAn Leeuwen, J. L. 2008 Flow patterns of larval fish: undulatory swimming in the intermediate flow regime. J. Expl Biol. 211 (2), 196-205.

van Rees, W. M., Gazzola, M. \& Koumoutsakos, P. 2013 Optimal shapes for intermediate Reynolds number anguilliform swimming. J. Fluid Mech. 722, R3.

TAYlor, G. K., NudDs, R. L. \& ThOmas, A. L. R. 2003 Flying and swimming animals cruise at a Strouhal number tuned for high power efficiency. Nature 425, 707-711.

TOKIC, G. \& YUE, D. K. P. 2012 Optimal shape and motion of undulatory swimming organisms. Proc. R. Soc. Lond. 279 (1740), 3065-3074.

Triantafyllou, G. S., Triantafyllou, M. S. \& Grosenbaugh, M. A. 1993 Optimal thrust development in oscillating foils with application to fish propulsion. J. Fluids Struct. 7, 205-224.

Triantafyllou, M. S. \& Triantafyllou, G. S. 1995 An efficient swimming. Sci. Am. 272 (3), 64-70.

Tytell, E. D., Borazjani, I., Sotiropoulos, F., Baker, T. V., Anderson, E. J. \& Lauder, G. V. 2010 Disentangling the functional roles of morphology and motion in the swimming of fish. Integr. Compar. Biol. 50 (6), 1140-1154.

WeBB, P. W. 1984 Body form, locomotion and foraging in aquatic vertebrates. Am. Zool. 24, $107-120$. 